\title{
FINANCIACIÓN PARA LA EMPRESA UTILIZANDO EL CAPM
}

\author{
Beatriz Herrera Garcia
}

Docente de la Facultad

\begin{abstract}
RESUMEN
Debido a la posibilidad de emitir títulos financieros, las empresas son capaces de obtener capital por diversas fuentes, así, los mercados permiten obtener una gran cantidad de recursos de una diversidad de inversores de forma que se facilite la posibilidad de emprender proyectos de envergadura. Los mercados de capitales, cargando implícitamente a las empresas diferentes precios, se convierten en un medio de asignar los recursos escasos entre diversos usos competitivos.
\end{abstract}

La eficiente toma de decisiones por parte de las empresas, se refiere a las ventajas de los recursos ajenos (deuda) sobre los recursos propios (acciones) a la hora de financiar inversiones. Las empresas tienen que financiar sus inversiones reales, y la forma habitual de hacerlo es emitit unos títulos (acciones, bonos, etc.) que los inversores individuales e institucionales compran. La propiedad de dichos titulos otorga unos derechos sobre recursos (inciertos) que generan en el futuro dichas inversiones reales.

Lo importante de ello es que al tomar la decisión en un momento dado, el inversor debe conocer,o más exactamente, tener una estimación racional - dada toda la información disponible- de lo que valen en ese preciso momento los derechos adquiridos. Esto se logra mediante los modelos de valoración de activos (en especial el CAPM), que permiten medir cuánto valen hoy los derechos adquiridos sobre los recursos futuros que generan las inversiones financieras. De esa manera, el mercado de capitales facilita a los inversores racionales su participación en el financiamiento de las empresas.

Palabras clave: Activos Arrow-Debreu, rendimiento esperado, riesgo (volatilidad).

\section{INTRODUCCIÓN}

Los conflictos de intereses que surgen entre los agentes económicos participantes en las empresas: accionistas, acreedores y gerentes (enfoque stakeholders) y el diseño óptimo de contratos que minimicen dichos conflictos se ha convertido en un área crucial de estudio dentro del problema caracterizado por la toma eficiente de decisiones.

Los inversores individuales y las empresas se enfrentan continuamente con la decisión de dónde invertir las rentas de que disponen con el objetivo de conseguir el mayor rendimiento posible al menor riesgo. Para determinar qué activos son atractivos para invertir y cuáles no, es decir, cuales son más rentables y cuales menos a igualdad de riesgo, los inversores necesitan un punto de referencia que les permita determinar cuándo un proyecto de inversión genera una rentabilidad superior y cuándo no. Esa rentabilidad es la tasa de rendimiento esperada, que se define como aquel rendimiento mínimo exigido por un inversor para realizar una inversión determinada.

El rendimiento esperado es aquel que el inversor en cuestión espera recibir a cambio de correr el riesgo de realizar una inversión 
determinada. Así, si el rendimiento esperado de un activo es inferior a la tasa de tendimiento requerida para invertir desechará su compra, mientras si se espera que ciertos activos proporcionen un rendimiento superior al requerido se recomendará su adquisición porque aumentará la riqueza del inversor.

Si un director de empresa -ejecutivo que corre con la responsabilidad de escoger entre posibles proyectos de inversión- requiere elegir los proyectos más rentables para los propietarios de la empresa, necesitará un punto de referencia con objeto de obtener una rentabilidad mínima a conseguir. Ese punto de referencia, es decir, esa mínima tasa de rendimiento requerida por la empresa es el coste de oportunidad del capital (o coste de capital) que indica aquella mínima tasa de rendimiento que permite a la empresa hacer frente al coste de los recursos financieros necesarios para acometer una inversión.

El coste de capital es la tasa de rendimiento interno que una empresa deberá pagar a los inversores para incitarlos a arriesgar su dinero en la compra de los títulos emitidos por ella; en otras palabras, es la mínima tasa de rentabilidad con la que deberá remunerar a las diversas fuentes financieras que componen su pasivo, con objeto de mantener a sus inversores satisfechos, evitando, al mismo tiempo, que descienda el valor de mercado de sus acciones. Tanto acreedores como accionistas de la empresa deben ser compensados por el coste de oportunidad de invertir sus fondos en un negocio en particular en lugar de otros con riesgo equivalente.

Por ello, el coste de capital es la tasa de rentabilidad que la empresa deberá conseguir con el objeto de satisfacer a los accionistas y acreedores por el nivel de riesgo que corren. Este es uno de los factores principales de la determinación del valor de la empresa y es utilizado como la tasa de descuento que actualiza la corriente de flujos de caja que la empresa promete generar.

\section{MARCO TEÓRICO}

Las empresas emiten activos financieros haciendo uso de los mercados de capitales. Los inversores individuales compran esos activos, apropiándose así de los derechos sobre los recursos que generan las inversiones reales que realizan las empresas. Así, las empresas reciben dinero para llevar a cabo sus inversiones reales. Finalmente, los inversores individuales obtienen los recursos generados por dichas inversiones. Sin embargo, las empresas pueden reinvertir parte o incluso la totalidad de los recursos generados siempre que dicha decisión maximice la riqueza del accionista que es, en definitiva, el objetivo último que debe buscar la empresa (Cuervo, A., et. aly Van Horne, et. al).

Los modelos de valoración de activos que se usan para calcular los precios de los activos no son más que un resumen de las secuencias anteriores y sirven para informar ¿cuánto valen hoy esos recursos que se generan en las inversiones reales y qué va a propiedad de los obligacionistas? La respuesta se obtiene a través del valor actual de la empresa y será el punto de referencia clave en la negociación de dichas acciones en los mercados de capitales.

Comolos componentes principales de la financiación empresarial son las acciones y las deudas, el objetivo es determinar la tasa mínima de rendimiento que la empresa deberá obtener en sus inversiones para satisfacer la tasa de rendimiento requerida por los inversores, es decir, para que la cotización de sus títulos en los mercados no descienda.

Para realizar este cálculo, será necesario tener en cuenta que el coste efectivo para 
la empresa de cada una de las principales fuentes financieras consistirá en averiguar la tasa de descuento que iguala lo efectivamente cobrado por la empresa, en el momento de emitir el título, con lo efectivamente pagado por la misma.

Por otro lado, si el objetivo de la empresa es maximizar la riqueza de sus accionistas (Mascareñas et.al), el equipo directivo debería retener los beneficios sólo si la inversión en dicha empresa fuese al menos tan atractiva como la mejor oportunidad de inversión que tengan dichos accionistas. En otro caso, si estos tienen unas oportunidades de inversión mejores, todos los beneficios deberían ser distribuidos vía dividendos. En resumen, la tasa de rendimiento requerida de los accionistas (coste de las acciones ordinarias) debería ser igual al rendimiento esperado de la mejor inversión disponible (inversión alternativa).

Por lo que los diversos modelos de valoración de activos bajo incertidumbre juegan continuamente con la interrelación entre carteras réplica y el concepto de diversificación, ambas ideas están íntimamente conectadas. Gracias a la capacidad de los inversores para construir carteras bien diversificadas, donde sólo cuenta el riesgo de mercado, resulta posible combinar dos fondos (al activo seguro y la cartera de mercado) con riesgo idéntico al activo que se desea valorar. Así, para evitar posibilidades de arbitraje, la cartera réplica y el activo a valorar deben tener el mismo rendimiento esperado. Estas ideas conducen al denominado "modelo de valoración de activos con cartera de mercado"-CAPM- ${ }^{1}$.

El CAPM -Capital Asset Pricing Model, es un modelo que se basa en una relación lineal entre rendimiento esperado y riesgo, éste modelo constituye una de las piezas fundamentales de las finanzas corporativas.

El CAPM fue desarrollado inicialmente por el Premio Nobel de Economía William Sharpe, parte de la base que la tasa de rendimiento requerida por un inversor es igual a la tasa de rendimiento sin riesgo más una prima de riesgo, donde el único riesgo importante en el riesgo sistemático.

1 Hay otros diversos modelos de valoración que miden el rendimiento esperado de la mejor inversión posible, entre ellos destacan los siguientes:

a) El conocido modelo de estructura de capital de Modigliani-Miller (ver, Modigliani, Franco y Miller, Merton, The Cost of Capital. Corporation Finance and the Theory of Investment, American Review 48, 1958), quienes suponen que el coste de capital medio ponderado y el valor de la empresa son totalmente independientes de la composición de la estructura del capital de la compañia, es decir, el valor de la empresa sólo dependerá de la capacidad generadora de renta de sus activos sin importar de dónde han procedido los recursos financieros que los han financiado.

b) El modelo de crecimiento de los dividendos o modelo de Gordon-Shapiro (ver, Gordon, M.J. y Shapiro, E., Capital Equipment Análisis: The Required Rate of Profit, Management Science, octubre de 1956), quienes parten del supuesto de que el precio teórico de una acción es igual al valor actual de los dividendos futuros que ello es capaz de proporcionar. A su vez, los dividendos crecerán a una tasa media constante y acumulativa por un tiempo indefinido.

c) El modelo de valoración a ttavés de atbitraje APT -Arbitraje Pricing Theory-o APM -Arbitraje Pricing Model-, desarrollado por Stephen Ross (ver, Finanqas Corporativas, 1995), es un modelo de equilibrio de cómo se determinan los precios de los activos financieros. Se basa en la idea de que en un mercado financiero competitivo, el arbitraje asegurará que los activos sin riesgo proporcionen el mismo rendimiento esperado. El modelo APT se basa en la idea de que los precios de los títulos se ajustan conforme los inversores construyen carteras de valores que persiguen la consecución de beneficios de arbitraje. Cuando ya no existan dichas oportunidades se alcanzará el equilibrio en los precios de los activos financieros. 
Como hipótesis de trabajo, se considera que un mercado ideal que jerarquiza las fuentes de financiación vendría representado por los llamados activos contingentes, también conocidos como activos Arrow-Debreu ${ }^{2}$. Un activo contingente es un activo que paga una unidad monetaria si un determinado escenario ocurre y nada, en caso contrario. La contingencia se asocia con la ocurrencia de un escenario concreto en el futuro, y así el precio del activo Arrow-Debreu nos proporciona el valor que tiene hoy una unidad monetaria recibida en el futuro en un escenario determinado. Esta teoría puede ayudar a explicar cómo las empresas de las economías emergentes pueden financiar con determinados recursos líquidos sus proyectos de inversión.

\section{RESULTADOS}

A los inversores sólo les preocupa el rendimiento esperado (su media) y la varian$\mathrm{za}^{3}$ de dichos rendimientos cuando se ven obligados a discriminar entre inversiones alternativas. Decir que sólo los inversores se preocupan del rendimiento esperado y de la varianza, implica suponer que la distribución de probabilidad de los rendimientos de los activos es normalo, alternativamente, que los inversores tienen una forma muy particular de preferencias e incluso una forma particular de aversión al riesgo.

La optimización de las inversiones en el contexto media-varianza, donde los agentes logran el máximo rendimiento esperado para un nivel de riesgo (varianza) dado, y que se debe al premio Nobel de Economía Harry Markowitz, se ha convertido en una de las herramientas que más aplicaciones ha tenido la toma de decisiones financieras. Ésta hace usos de los conceptos de ausencia de arbitra$\mathrm{je}^{4}$ y carteras réplica, lo que implica valorar activos financieros en relación siempre a otros activos.

El rendimiento esperado de cualquier activo puede replicarse mediante una cartera que combina exclusivamente dos fondos o carteras. Una de ellas será un activo seguro, activo que permita prestaro pedir prestado al tipo de interés libre de riesgo, mientras que el segundo fondo consiste en la denominada cartera de mercado, que incorpora todos los activos existentes en proporción cada uno de ellos a su valor de mercado o capitalización (precio del activo por número de accione so títulos desembolsados que posee la empresa). Esta cartera réplica se construye de manera que tenga riesgo idéntico al activo que se desea valorar.

2 Kenneth Arroz, Gerard Debreu y Franco Modigliani fueron galardonados con el Premio Nobel de Economía por sentar las bases de lo que actualmente se conoce como finanzas modernas.

3 La varianza se describe como la variabilidad que experimentan los rendimientos (precios) de un activo alrededor de su media. Su expresión es:

$$
\sigma^{2}=\mathrm{E}\left[\mathrm{R}_{\mathrm{i}}-\mathrm{E}\left(\mathrm{R}_{\mathrm{j}}\right)\right]^{2}=\sum_{j=1}^{s} \pi \mathrm{s}\left[\mathrm{R}_{\mathrm{j}}-\mathrm{E}\left(\mathrm{R}_{\mathrm{j}}\right]^{2}\right. \text {. }
$$

4 Modigliani y Miller introdujeron el concepto de arbitraje, el cual significa beneficiarse de la diferencia de valor existente en dos mercados distintos con respecto a un mismo bien, es decir, cualquier estrategia de inversión que permita ganar dinero a cambio de nada es una estrategia de arbitraje. Lo contrario sucede bajo ausencia de arbitraje, es decir, debe ser cierto que si un activo financiero o una combinación de activos (cartera) produce pagos (flujos de caja) futuros no negativos, entonces, necesariamente el coste de dicho activo o cartera en el momento de realizar la inversión debe ser también no negativo. 
La diversificación del riesgo significa que los agentes económicos son capaces de eliminar parte del riesgo (varianza) de sus inversiones combinando los activos disponibles (formando carteras) en el mercado. Únicamente, aquella parte del riesgo que no puede eliminarse mediante la diversificación será relevante. Esta parte del riesgo está directamente asociada al riesgo global de la economía por lo que suele denominarse riesgo de mercado (es el denominado riesgo sistemático).
Es de advertir, que el resultado de la varianza (medida de dispersión) no está representada en la misma unidad de medida que la variable rendimiento, por lo que debe obtenerse una medida de igual significado y que se deriva de la varianza, la denominada desviación estándar $(\sigma)$ o volatilidad del rendimiento.

Para la estimación de los rendimientos y su volatilidad, se utilizará el siguiente procedimiento:

\section{Ejemplo 1:}

Suponga que en un determinado mercado se registra la siguiente información acerca de los rendimientos de tres activos inciertos en tres escenarios (s) distintos: buena (B), regular (R) y mala (M).

\begin{tabular}{|c|}
\hline Situación previsi- \\
ble del mercado \\
$\mathrm{B}$ \\
$\mathrm{R}$ \\
$\mathrm{M}$ \\
\hline
\end{tabular}

\begin{tabular}{|c|}
\hline Probabilidad de \\
los escenarios $(\pi)$ \\
$25 \%$ \\
$50 \%$ \\
$25 \%$ \\
\hline
\end{tabular}

\begin{tabular}{|c|}
\hline Rendimiento del \\
activo $1(\%)$ \\
14 \\
8 \\
4 \\
\hline
\end{tabular}

\begin{tabular}{|c|}
\hline Rendimiento del \\
activo 2 (5) \\
17 \\
9 \\
6 \\
\hline
\end{tabular}

\begin{tabular}{|c|}
\hline Rendimiento del \\
activo $3(\%)$ \\
3 \\
9 \\
15 \\
\hline
\end{tabular}

Con esa información, se pide calcular los rendimientos esperados ${ }^{5}$ para cada activo en particular y sus correspondientes volatilidades.

\section{Solución}

El rendimiento esperado de los activos, $\left[E\left(R_{p}\right)\right]$, se calcula utilizando la siguiente expresión:

$E\left(R_{1}\right)=\pi_{i} R_{11}+\pi_{i} R_{i 2}+R_{i 3}$

Activo 1: $\mathrm{E}\left(\mathrm{R}_{1}\right)=0.25(0.14)+0.50(0.08)+0.25(0.04)=8.5 \%$

Activo 2: $\left.\left.\mathrm{E}\left(\mathrm{R}_{2}\right)=0.25(0.17)+0.50(0.09)+0.25\right) 0.06\right)=10.25 \%$

Activo 3: $\mathrm{E}\left(\mathrm{R}_{3}\right)=0.25(0.03)+0.50(0.09)+0.25(0.015)=9.0 \%$

5 El rendimiento esperado, $E(R)$, de una cartera es la media ponderada de los rendimientos esperados de los componentes de dicha cartera de activos. 
Varianza y desviación estándar (volatilidad) de los activos

\begin{tabular}{|c|c|c|c|c|}
\hline Escenario & {$\left[R_{i s}-E\left(R_{j}\right)\right]$} & {$\left[R_{i s}-E\left(R_{j}\right)\right]^{2}$} & $\pi_{s}\left[R_{s}-E(R)\right]^{2}$ & Desv. Estándar \\
\hline B & $14-8.5=5.5$ & 30.25 & 75625 & \\
\hline $\mathrm{R}$ & $8-8.5=0.5$ & 0.25 & 0.1250 & \\
\hline M & $4.8 .5=4.5$ & 20.25 & $\begin{array}{c}5.0625 \\
\sigma^{2}=12.75 \sigma\end{array}$ & $\sigma_{1}=3.57 \%$ \\
\hline Activo2: & & & & \\
\hline B & $17-10.25=6.75$ & 45.56 & 11.39 & \\
\hline $\mathrm{R}$ & $9-10.25=1.25$ & 1.56 & 0.78 & \\
\hline M & $6-10.25=4.25$ & 18.56 & $\begin{array}{c}4.51 \\
\sigma^{2}=16.68\end{array}$ & $\sigma_{2}=4.08 \%$ \\
\hline Activo 3: & & & & \\
\hline B & $3-9.0=-6$ & 36 & 9 & \\
\hline $\mathrm{R}$ & $9-9.0=0$ & 0 & 0 & \\
\hline $\mathrm{M}$ & $15-9.0=6$ & 36 & $\begin{array}{c}9 \\
\sigma_{3}^{2}=18\end{array}$ & $\sigma_{3}=4.24 \%$ \\
\hline
\end{tabular}

En el caso del rendimiento esperado de los activos y su medida de dispersión (volatilidad) que mira hacia el futuro, se tienen los siguientes resultados:

$\begin{array}{lll} & \mathrm{E}\left(\mathrm{R}_{\mathrm{j}}\right) & \sigma_{\mathrm{j}} \\ \text { Activo } 1 & 8.5 \% & 3.57 \% \\ \text { Activo } 2 & 10.25 \% & 4.08 \% \\ \text { Activo 3 } & 9.0 \% & 4.24 \%\end{array}$

En cambio, la covarianza es una medida de cómo los rendimientos de los activos tienden a moverse conjuntamente. Es una medida que juega un papel importante en la valoración de los activos financieros. A diferencia de la varianza que nunca puede ser negativa, la covarianza puede serlo cuando los rendimientos de los activos tienden a moverse en direcciones opuestas.

Resulta útil normalizar la covarianza de de forma que acotemos sus valores entre -1 y
+1. Para ello, basta dividir la covarianza entre el producto de las desviaciones estándar de los activos y se obtiene el denominado "coeficiente de correlación”. Así, la covarianza puede escribirse en términos de coeficiente de correlación como:

$$
\operatorname{cov}\left(R_{1}, R_{2}\right) \equiv \sigma_{12}=\rho_{12} \sigma_{1} \sigma_{2}
$$

Donde: $\rho_{12}$ es el coeficiente de correlación entre el rendimiento del activo 1 y el activo2. Por otro lado, se sabe que $-1 \leq \rho_{12} \leq 1$. Estas cotas permiten saber con precisión el grado de variación conjunta que experimentan los rendimientos de dos activos financieros.

\section{Ejemplo 2:}

Utilizando los datos y resultados del ejemplo anterior, se procede a determinar la covarianza y las correlaciones entre los tres activos financieros. En resumen, los resultados obtenidos son los siguientes:

\begin{tabular}{|c|c|c|c|c|}
\hline Escenario & $\pi_{s}$ & {$\left[\mathrm{R}_{1}-\mathrm{E}\left(\mathrm{R}_{1}\right)\right]$} & {$\left[\mathrm{R}_{2}-\mathrm{E}\left(\mathrm{R}_{2}\right)\right]$} & $(3)$ \\
& $(1)$ & $(2)$ & 6.75 & $(4)$ \\
$\mathrm{B}$ & $25 \%$ & 5.5 & -1.25 & 6.0 \\
$\mathrm{R}$ & $50 \%$ & -0.5 & -4.25 & 0 \\
$\mathrm{M}$ & $25 \%$ & -4.5 & 6.0 \\
\hline
\end{tabular}


Los cálculos del coeficiente de correlación entre los activos 1,2 y 3 se efectúan después de obtener las covarianzas $\left(\sigma_{12}\right)$ correspondientes.
Donde: $\mathrm{X}_{\mathrm{j}}$ es el pago en unidades monetarias del activo $j$ en el escenario s. $\phi_{s}$ es el precio (valor) del activo Arrow-Debreu, que

\begin{tabular}{|c|c|c|}
\hline $\begin{array}{l}\text { Entre activos } 1 \text { y } 2 \\
(1)(2)(3)\end{array}$ & $\begin{array}{c}\text { Entre activos } 1 \text { y } 3 \\
(1)(2)(4)\end{array}$ & $\begin{array}{c}\text { Entre activos } 2 \text { y } 3 \\
\text { (1)(3)(4) }\end{array}$ \\
\hline 9.2813 & -8.25 & -10.1250 \\
\hline 0.3125 & 0.00 & 0.00 \\
\hline$\sigma_{12}=\sum_{s=1}^{4.7813}=14.3751$ & $\sigma_{13}=\sum_{s=1}^{-6.25}=-15.0$ & $\sigma_{23}=\sum_{s=1}^{-6.3750}=-16.5$ \\
\hline
\end{tabular}

Dividiendo las covarianzas entre el producto de las desviaciones estándar de los activos relacionados se obtienen los coeficientes de correlación, como sigue:

$\rho_{12}=\sigma_{12} /\left(\sigma_{1}\right)\left(\sigma_{2}\right)=\frac{14.3751}{(3.5707)(4.0853)}$

$=0.9854$

$\rho_{13}=\sigma_{13} /\left(\sigma_{1}\right)\left(\sigma_{3}\right)=\frac{-15.0}{(3.5707)(4.2426)}$

$=-0.9902$

$\rho_{23}=\sigma_{23} /\left(\sigma_{2}\right)\left(\sigma_{3}\right)=\frac{-16.5}{(4.0853)(4.2426)}$

$=-0.9520$

Análisis de los resultados. En el caso del activo 1 y del activo 2 , se puede observar que ambos están positivamente correlacionados. De hecho el coeficiente de correlación (0.9854) es elevadísimo, es decir, están (casi) perfectamente correlacionados. En los otros dos casos, el activo 3 está (casi) perfecta y negativamente correlacionado con el activo 1 y el activo 2 .

\section{Laecuaciónfundamental devaloración} y el papel de los Activos Arrow-Debreu.

La ecuación fundamental de valoración de activos está dada por la expresión:

$$
\mathrm{V}_{\mathrm{j}}=\sum_{s=1}^{s} \phi_{\mathrm{s}} \mathrm{X}_{\mathrm{js}} \circ \mathrm{P}_{\mathrm{i}}=\sum_{s=1}^{s} \phi_{\mathrm{s}} \mathrm{X}_{\mathrm{is}}
$$

paga una unidad monetaria en el escenarios y nada en caso contrario.

Esta expresión nos dice que el precio o valor de cualquier activo $j$, consistente con la ausencia de arbitraje, es el valor actual de sus pagos (flujos de caja) futuros, donde los factores de descuento reflejan tanto la incertidumbre de cada escenario donde se generan los flujos de caja como el valor temporal del dinero $y$, vienen recogidos por los precios de los activos A.D.

La ecuación anterior puede modificarse al introducir las probabilidades verdaderas $\left(\pi_{\mathrm{s}} ;\right.$ para $\left.\mathrm{s}=1, \ldots, \mathrm{s}\right)$.

$$
\mathrm{P}_{\mathrm{j}}=\sum_{s=1}^{s} \phi_{s} \mathrm{X}_{\mathrm{js}}=\sum_{s=1}^{s} \pi_{s}\left[\begin{array}{c}
\phi s \\
\pi s
\end{array}\right] \mathrm{X}_{\mathrm{j} s}
$$

Donde: $\left[\begin{array}{l}\phi s \\ \pi s\end{array}\right]=\mathrm{M}_{\mathrm{s}}$ es interpretada como el precio del activo A-D s-ésimo por unidad de probabilidad verdadera del propio escenarios.

Por tanto, la ecuación fundamental de valoración puede reescribirse como:

$$
\mathrm{Pj}=\sum_{s=1}^{s} \pi_{s} \mathrm{M}_{s} \mathrm{X}_{\mathrm{is}}=\mathrm{E}[\mathrm{M} \mathrm{X} \mathrm{j}] ; 1, \ldots, \mathrm{N}
$$


Así, el precio de cualquier activo financiero jes el valor esperado (E) bajo la verdadera probabilidad $\pi_{\mathrm{s}}$, de sus pagos of flujos de caja futuros ponderados por una variable agregada. Dicha variable agregada $M$ debe ser tanto un factor de descuento como una variable que pondere los flujos generados por $j$, según sea el escenario donde se reciben.

La aplicación del modelo de valoración de activos y el papel de los activos A-D, se pueden apreciar en el siguiente caso.

\section{Ejemplo 3:}

Suponga que una compañía esta considerando la posibilidad de explotar una mina de cobre y que la fuente básica de incertidumbre es el precio que el cobre tendrá en los mercados internacionales. Si los países productores de cobre (agrupados en el CIPEC), se ponen de acuerdo y fijan una política concreta de extracción del cobre, su precio alcanzaría $\$ 0.28$ / lb. (dólares por libra) en un año y la mina tendría un valor de 100 millones de dólares. En caso contrario, el precio se quedaría en $\$ 0.23 / \mathrm{lb}$., dentro de un año, y la mina valdría 25 millones de dólares. Se supone, además, que el cobre puede almacenarse sin costo. El precio del cobre hoy es igual a $\$ 0.25 / \mathrm{lb}$., y que el tipo de interés del activo seguro a un año es igual a $5.82 \%$-nótese que el precio del bono básico hoy sería igual a $(1 / 1+r)=1 / 1.0582$ $=0.945$ dólares - .

Presentación de los datos:
La interrogante para la compañía es conocer el valor de la mina de cobre hoy.

El procedimiento de solución en éste caso es utilizar la herramienta de valoración de activos bajo incertidumbre, que se basa en la construcción (formación) de una cartera de activos existentes que replique los pagos futuros de la mina (activo a valorar).

La mina de cobre es un activo contingente cuyo valor depende o es contingente con el valor futuro del cobre en el mercado internacional. $\mathrm{El}$ cobre es un activo que se puede negociar en los mercados internacionales. La fuente de incertidumbre asociada al activo (real) a valorar y el subyacente (el activo subyacente es el cobre) es la misma $\mathrm{y}$, además, se tienen dos escenarios futuros $(\mathrm{s}=1$ y s=2). Finalmente, existe también la posibilidad de invertir en un activo seguro (el bono básico).

Generalizando el ejemplo, se puede obtener una técnica de valoración de activos bajo incertidumbre, basada en el supuesto fundamental de ausencia de arbitraje (esta técnica es clave en las finanzas y su aplicación incluye, prácticamente, todos los modelos de valoración de activos - se trata de replicar los pagos de un activo en cada escenario futuro-).

Un activo contingente o activo A-D es un activo que paga una unidad monetaria si un determinado escenario ocurre y nada en caso

\begin{tabular}{|c|c|c|c|}
\hline Escenarios & Hoy & $\begin{array}{l}\text { En un año } \\
\text { Precio alto }(s=1)\end{array}$ & $\begin{array}{l}\text { En un año } \\
\text { Precio bajo }(s=2)\end{array}$ \\
\hline Precio del cobre & $\$ 0.25 / \mathrm{lb}$ & $\$ 0.28 / \mathrm{lb}$ & $\$ 0.23 / \mathrm{lb}$ \\
\hline Valor mina de cobre & & 100 mill.\$ & 25 mill.\$ \\
\hline Valor bono básico & $\$ 0.945$ & 1 dólar & 1 dólar \\
\hline
\end{tabular}


contrario. El concepto de activo A-D no es más que la extensión del concepto de bono básico al caso en que existe incertidumbre caracterizada por la ocurrencia de un escenario concreto en el futuro $y$, así, el precio del activo A-D nos proporciona el valor que tiene hoy un dólar recibido en el futuro en un escenario determinado.

Como en el ejemplo existen dos escenarios, por lo tanto, deben existir dos activos A-D. El activo 1 paga un dólar en el escenario $\mathrm{s}=1 \mathrm{y}$ nada en el escenario $\mathrm{s}=2$. El activo 2 paga un dólar en el escenario $\mathrm{s}=2 \mathrm{y}$ cero en el escenatio $s=1$. Se denomina como $\phi_{\mathrm{s}}$ al precio hoy del activo A-D que paga un dólar en el escenario s y nada en caso contrario.

En consecuencia, si se quiere replicar los valores futuros del cobre mediante activos A-D, se debería mantener una cartera con 0.28 títulos A-D \#1 y 0.23 títulos del activo A-D \#2.

Dada la definición de los activos A-D, los pagos de esta cartera en cada escenario serían:

\begin{tabular}{|c|c|c|c|c|c|}
\hline $\begin{array}{l}\text { Activ } \\
\# 1\end{array}$ & A-D & $\begin{array}{c}\text { Activo A-D } \\
\quad \# 2\end{array}$ & & & \\
\hline 28 & $x$ & 0.23 & 0 & $=$ & $\$ 0.28$ \\
\hline & 0 & 0.23 & $+\quad 1$ & $=$ & $\$ 0.23$ \\
\hline
\end{tabular}

Evitando las posibilidades de arbitraje, el coste hoy de esta cartera de activos A-D debe ser igual al valor hoy del cobre (ecuación 1):

Coste hoy cartera de activos A-D Coste hoy del cobre

$$
0.28 \phi_{1}+0.23 \phi_{2}=\$ 0.25
$$

Donde: $\phi_{1}$ es el precio hoy del activo AD \# 1 y, $\phi_{2}$ es el precio hoy del activo A-D \#2.

Igualmente, los pagos del activo seguro pueden replicarse manteniendo una cartera compuesta de un título de cada activo A-D existente:
Activo A-D Activo A-D

\#1 \#2

$1 \times 1+1 \times 0=1$ dólar

$1 \times 0+1 \times 1=1$ dólar

Nuevamente, para evitar arbitraje, el coste de la cartera de activos A-D debe ser igual al coste del activo seguro, formándose la siguiente ecuación 2 :

Coste hoy cartera activos A-D Coste del bono básico

$$
1 \phi_{1}+1 \phi_{2}=0.945
$$

Relacionando las ecuaciones (1) y (2) de valoración, se observa que se trata de un sistema de dos ecuaciones (una para cada activo negociado en el mercado), y dos incógnitas (los precios de los activos $A-D$ ).

$$
\begin{aligned}
& 0.28 \phi_{1}+0.23 \phi_{2}=0.25 \\
& \phi_{1}+\phi_{2}=0.945
\end{aligned}
$$

Resolviendo las ecuaciones con dos incógnitas, se obtienen los valores (precios) de los activos A-D.

$$
\phi_{1}=0.653 \quad \text { y } \phi_{2}=0.292
$$

Con estos precios de los activos A-D se puede valorar cualquier activo financiero. De hecho, estos precios son pieza clave en toda expresión de valoración de activos financieros. Reflejan lo que los inversores están dispuestos a pagar hoy por unidades de consumo en cada uno de los escenarios futuros. Recogen, por tanto, la incertidumbre asociada a cada uno de dichos escenarios y son, además, precios de hoy, por lo que incorporan un factor de descuento temporal.

Mediante la utilización de los valores de los activos A-D se puede obtener el valor de la mina de cobre (el activo a valorar):

$$
\begin{aligned}
& \mathrm{V}=100 \mathrm{f}_{1}+25 \mathrm{f}_{2} \\
& \mathrm{~V}=100(0.653)+25(0.292) \\
& \mathrm{V}=72.6 \text { millones de dólares. }
\end{aligned}
$$


En la ecuación, 100 y 25 son los flujos de caja que genera la mina de cobre en cada uno de los escenarios futuros.

A manera de conclusión, repetimos que los precios de los activos A-D pueden utilizarse para valorar cualquier activo (real o financiero) cuyos pagos (flujos de caja) pueden especificarse en cada escenario.

\section{ANÁLISIS Y DISCUSIÓN}

ElCAPM o modelo de valoración de activos con cartera de mercado bajo incertidumbre que fue rescrita anteriormente como:

$\mathrm{P}_{\mathrm{i}}=\sum_{s=1}^{s} \pi_{\mathrm{s}} \mathrm{M}_{\mathrm{s}} \mathrm{X}_{\mathrm{js}}=\mathrm{E}\left[\mathrm{MX} \mathrm{X}_{\mathrm{j}}\right] ;_{\mathrm{j}=1, \ldots, \mathrm{N}}$

Esta expresión noes más que la ecuación fundamental de valoración bajo ausencia de arbitraje, que suele utilizarse en los análisis empíricos sobre los mercados financieros. Hasta ahora, las ecuaciones de valoración han sido presentadas en términos de precios de los activos financieros, sin embargo, en las finanzas modernas se suele utilizar tasas de rendimiento esperado en lugar de precios para describir las relaciones fundamentales existentes entre riesgo y rendimiento.

Por lo que la ecuación anterior se puede expresar en términos de tasas de rendimiento, dividiéndola cada lado de la ecuación por el precio del activo $\mathfrak{j}$, resultando:

$1=\sum_{s=1}^{s} \phi_{\mathrm{s}} \bar{R}_{\mathrm{js}}=\sum_{s=1}^{s} \pi_{\mathrm{M}} \bar{R}_{\mathrm{js}}=\mathrm{E}\left[\mathrm{M} \bar{R}_{\mathrm{j}}\right] ;{ }_{\mathrm{j}=1, \ldots, \mathrm{N}}$

Donde: $\bar{R}_{\text {js }}$ es el rendimiento bruto obtenido por el activo j, si ocurre el escenario s. Por tanto, $\bar{R}_{j}$ es $1+$ la tasa porcentual de rendimiento de $R_{i}$. Recuérdese que $M_{s}=\phi_{s} / \pi_{s}$.
Esta expresión indica que los rendimientos esperados de todos los activos deben ser iguales. Dice que dichos rendimientos esperados son iguales una vez ponderados por la variable agregada $M$. Dado que $M$ refleja la importancia que tiene recibir flujos de caja en uno u otro escenario, y dado que los activos financieros tenderán a pagar o generar distintos flujos de caja se convierte en distintos escenarios y, la ponderación de los rendimientos o de los flujos de caja se convierte en la variable clave para comprender la valoración de los activos.

Tanto las propiedades estadísticas que caracterizan los rendimientos de los activos, como la transformación de la ecuación fundamental en una ecuación que permita relacionar de manera muy intuitiva el rendimiento esperado y el riesgo para cada activo, aconseja el empleo de la ecuación fundamental de valoración en términos de rendimientos (tasas de rendimiento) en lugar de precios. Así, la ecuación fundamental de valoración expresada en tasas de rendimiento, tiene la siguiente expresión simplificada:

$\mathrm{E}\left(\mathrm{M} \bar{R}_{\mathrm{p}}\right)=\mathrm{E}(\mathrm{M}) \mathrm{E}\left(\bar{R}_{\mathrm{j}}\right)+\operatorname{cov}\left(\mathrm{M}, \mathrm{R}_{\mathrm{j}}\right)=1$

Esta expresión permite obtener una fórmula de valoración en términos del rendimiento esperado de cualquier activo incierto j. Así, el rendimiento esperado del activo incierto j es:

$\mathrm{E}\left(\bar{R}_{\mathrm{j}}\right)={ }_{E(M)}^{1}-\frac{\operatorname{cov}(M, R j)}{E(M)}=1+\mathrm{r}-\frac{\operatorname{cov}(M, R j)}{E(M)}$

Donde: $\mathrm{E}(\mathrm{M})=\frac{1}{(1+r)^{\prime}}$, donde $r$ es el tipo de interés del activo libre de riesgo.

Asimismo, bajo la ecuación fundamental de valoración y ausencia de arbitraje la prima 
de riesgo ${ }^{6}$ de cualquier activo incierto $j$, que está definida por el exceso de rendimiento esperado entre el activo $j$ y el activo libre de riesgo viene dado por:

$$
E\left(\mathrm{R}_{\mathrm{i}}-\mathrm{r}\right)=-\left[\begin{array}{c}
1 \\
E(M)
\end{array}\right] \operatorname{cov}\left(\mathrm{M}, \mathrm{R}_{\mathrm{j}}\right)
$$

Esta ecuación está escrita en términos de prima de riesgo del activo $j$, donde $R_{i}$ hace ahora el papel de tasa porcentual de rendimiento al haberse cancelado los años asociados al rendimiento bruto del activo $j$, y del activo seguro.

Todo activo incierto debe tener una prima de riesgo positiva. Formalmente, cualquier activo que tenga una prima de riesgo distinta de cero es un activo incierto. Por lo que ningún inversor estaría dispuesto a arriesgar sus recursos en un activo incierto si estima un rendimiento menor que el tipo de interés que ofrece el activo libre de riesgo.

En definitiva, dos aspectos claves deben destacarse en la ecuación de valoración anterior:

a) Dicha expresión no es más que una relación entre rendimiento esperado y riesgo que deben satisfacer todos los activos financieros. Esta interpretación implica que la forma apropiada de medir el riesgo de los activos financieros individuales es la covarianza, no la desviación estándat (varianza de sus rendimientos). Esta idea sugiere que la covarianza como medida de riesgo individual es un concepto absolutamente general, y no relacionado con los supuestos del modelo media-varianza.

b) Es importante analizar la variable agregada $M$ para discutir con detalle que determina la prima de riesgo de los activos financieros. Lo que se sabe es que el factor determinante de la prima de riesgo de cualquier activo es la cov $\left(M, R_{\mathrm{i}}\right)$. En el CPM se parte de la base de que la tasa de rendimiento requerida de un inversor es igual a la tasa de rendimiento sin riesgo más una prima de riesgo, donde el único riego importante es el riesgo sistemático (riesgo de mercado). Este nos indica cómo responde el rendimiento de la acción ante las variaciones sufridas en el rendimiento del mercado bursátil, dicho riesgo se mide a través del coeficiente de volatilidad conocido como beta $(\boldsymbol{\beta})^{7}$.

\section{CONCLUSIONES}

En suma, las expresiones: $\mathrm{E}\left(\mathrm{M}, R_{\mathrm{i}}\right)=1$ y $E\left(\mathrm{R}_{\mathrm{i}}-\mathrm{r}\right)=-\left[\begin{array}{c}1 \\ E(M)\end{array}\right] \operatorname{cov}\left(\mathrm{M}, \mathrm{R}_{\mathrm{i}}\right)$, nos permiten ofrecer un contenido compacto y bien definido de los que se entiende por economía financiera, tanto desde el punto de vista de los mercados como de la propia empresa. Cualquier otro modelo de valoración es, simplemente un caso especial.

6 La prima de riesgo mide el grado de aversión al riesgo que soporta determinado mercado bursátil al reflejar lo que los inversores exigen, en promedio, sobre el tipo de interés libre de riesgo para invertir en renta variable. Prima de riesgo $=E\left(R_{m}\right)-r$ ], donde $R_{m}$ es el rendimiento esperado del mercado (bursátil) y r es el rendimiento libre de riesgo de la inversión en el bono cupón cero representado pot los bonos del Tesoro a un año.

7 Beta mide la variación del rendimiento de un título con respecto a la variación del rendimiento del mercado. $E$ : cálculo de beta se tealiza a través de una regresión simple entre el rendimiento del título, o cartera, y us rend:mientos del índice butsátil tomado como referencia. La tangente del ángulo de la recta de regresión es el valor de beta. La relación entre rendimiento esperado y beta se puede expresar como: $E\left(R_{j}\right)=r+\left[E\left(R_{r}\right)-r\right] \beta_{i r}$ 
Ahora bien, en el modelo de valoración de activos financieros inciertos con cartera de mercado-CAPM-, interesa saber cuál será la única cartera eficiente de activos inciertos que mantendrán todos los inversores independientemente de si existe o no un activo seguro. En ambas situaciones (con o sin activo seguro), la cartera apropiada será la denominada "cartera de mercado", que pondera a cada activo financiero existente en el mercado según su capitalización bursátil (es el precio del mercado del activo por el número de acciones que tiene la empresa en circulación en el mercado).

William Sharpe ${ }^{8}$ y J. Lintner fueron los economistas que, trabajando individualmente, demostraron el modelo de cartera de mercado como cartera tangente ${ }^{9}$ (cartera eficiente para cualquier inversor cuando existe un activo seguro).

El CAPM es, de hecho, un modelo de equilibrio (el supuesto de la no existencia de oportunidades de arbitraje es una condición necesaria para obtener el equilibrio), y es un modelo estático, donde todos los inversores escogen sus carteras exclusivamente según el rendimiento esperado y varianza (volatilizada) de las mismas, lo que implica que la distribución de probabilidades delos rendimientos se especifica completamente por su media y varianza. En particular, el modelo suponeque los rendimientos de los activos se distribuyen como una variable normal.
Por otro lado, como todos los inversores combinan los activos inciertos en la misma proporción, debido a que todos ellos invertirán en la única cartera eficiente de activos inciertos, que es óptima, dado un activo seguto. Por lo que en le modelo, el único activo (cartera) incierto que se demanda es la cartera tangente. Así, dicha cartera debe ser necesariamente la cartera de todos los activos existentes en el mercado, donde cada uno de ellos esté en proporción a su valor de mercado o capitalización. En definitiva, la cartera tangente de activos inciertos debe ser, necesariamente, la cartera de mercado.

Cabe señalar que la coincidencia entre la cartera tangente de activos inciertos, cuando existe un activo seguro, y la cartera de mercado, implica que se produzca el denominado vaciado de mercado o que, en otras palabras, la oferta total (agregada) de activos financieros, que se supone exógena en el modelo, sea igual a la demanda total (agregada) de activos.

La formación de una cartera tangente de manera empírica puede ser obtenida ser obtenida siguiendo el procedimiento a continuación.

\section{Ejemplo 4:}

A finales de diciembre de 2002, el mercado financiero estaba compuesto únicamente de cuatro activos cuyos precios y capitalización bursátil se registra a continuación:

8 Sharpe, W., Alexander, G. yJ. Bailey, Investment, Prentice Hall International, 1995. La concesión en 1990 del Premio Nobel de Economía a H. Markowitz, M. Millar y W. Sharpe, representó el reconocimiento a la especialidad de las finanzas.

- Es la recta de mayor pendiente posible dado el conjunto de oportunidades de inversión e $N$ activos inciertos y el activo seguro. Dicha recta es la nueva frontera de carteras eficientes, donde T es la cartera de activos inciertos situada en la recta tangente a la frontera de carteras eficientes. 
Cuadro 1

\begin{tabular}{lcccc}
\hline Empresas & $\begin{array}{c}\text { Precio en } \\
\text { dólares (1) }\end{array}$ & $\begin{array}{c}\text { Acciones en cir- } \\
\text { culación en } \\
\text { millones (2) }\end{array}$ & $\begin{array}{c}\text { Capitalización en } \\
\text { mill. dólares } \\
\mathbf{( 3 )}=(\mathbf{1})(\mathbf{2})\end{array}$ & $\begin{array}{c}\text { Porcentaje del mer- } \\
\text { cado } \\
\mathbf{( 4 )}=(\text { Tot.3) } /(\mathbf{3})\end{array}$ \\
\hline Banco X & 79.35 & 100.77 & 7.996 & 21.44 \\
Empresa 1 & 23.60 & 891.54 & 21.040 & 56.42 \\
Empresa 2 & 28.17 & 174.09 & 4.904 & 13.15 \\
Empresa 3 & 21.63 & 162.23 & 3.509 & 9.40 \\
TOTAL & & $\mathbf{1 , 3 2 8 . 6 3}$ & 37.291 & 100. \\
\hline
\end{tabular}

Ahora, suponga que en éste mercado sólo existen tres inversores (los individuos: Pedro, Julio y Marcelo), cuya riqueza (capital) está distribuida de la forma siguiente:

Cuadro 2

\begin{tabular}{ccc}
\hline Inversores & $\begin{array}{c}\text { Riqueza en el } \\
\text { mercado } \\
\text { (En mill. dólares) }\end{array}$ & $\begin{array}{c}\text { Porcentaje de } \\
\text { riqueza del } \\
\text { mercado }\end{array}$ \\
\hline $\mathrm{P}$ & 13.491 & 47.69 \\
$\mathrm{~J}$ & 11.200 & 39.59 \\
$\mathrm{M}$ & 3.600 & 12.72 \\
TOTAL & $\mathbf{2 8 . 2 9 1}$ & $\mathbf{1 0 0 . 0 0}$ \\
\hline
\end{tabular}

Según el modelo (el CAPM), todos los inversores individuales invierten en la misma cartera tangente de activos inciertos. Esto significa que Pedro, Julio y Marcelo de éste mercado tendrian una cartera idéntica (en porcentaje) de activos inciertos.

El tamaño de la cartera de cada inversor no es el mismo, sino que cada inversor debe tener un número de acciones de cada una de las cuatro empresas que coincida con el porcentaje del mercado que posee. Esto significa que Pedro debería tener un 47.69\% de los títulos de las cuatro carteras existentes (ver, columna 3 del cuadro 2), Julio el $39.59 \%$ y Marcelo el $12.72 \%$. La consecuencia de estos porcentajes es que el $21.44 \%$ del valor de mercado de la cartera de cada inversor corresponde al Banco X (ver, columna 4 del cuadro 1), el $56.59 \%$ a la Empresa 1, el $13.15 \%$ a la Empresa 2 y el $9.40 \%$ a la Empresa 3.

Cuadro 3. Formación de la cartera de mercado

\begin{tabular}{lcccccccc}
\hline Empresa & \multicolumn{2}{c}{$\begin{array}{c}\text { Pedro } \\
\text { Acciones Valor }\end{array}$} & \multicolumn{2}{c}{$\begin{array}{c}\text { Julio } \\
\text { Acciones Valor }\end{array}$} & \multicolumn{2}{c}{$\begin{array}{c}\text { Marcelo } \\
\text { Acciones Valor }\end{array}$} & \multicolumn{2}{c}{$\begin{array}{c}\text { Total } \\
\text { Acciones Valor }\end{array}$} \\
\hline Banco X* & 48.06 & $3,813.56$ & 89.89 & $3,165.27$ & 82.82 & $1,017.26$ & 77.77 & $7,996.09$ \\
Empresa 1 & 17.17 & $10,034.01$ & 96.96 & $8,329.85$ & 40.40 & $2,676.24$ & 54.54 & $21,040.34$ \\
Empresa 2 & 2.02 & $2,338.67$ & 92.92 & $1,941.47$ & 14.14 & 623.68 & 9.09 & $4,904.11$ \\
Empresa 3** & 77.37 & $1,673.51$ & 23.23 & $1,389.29$ & 63.63 & 446.23 & 23.23 & $3,509.03$ \\
TOTAL & $\mathbf{6 3 3 . 6 2}$ & $\mathbf{1 7 , 8 5 9 . 7 5}$ & $\mathbf{5 2 6}$ & $\mathbf{1 4 , 8 2 5 . 8 8}$ & $\mathbf{1 6 8 . 9 9}$ & $\mathbf{4 , 7 6 6 . 4 1}$ & $\mathbf{1 , 3 2 8 . 6 3}$ & $\mathbf{3 7 , 4 4 9 . 5 7}$ \\
\hline
\end{tabular}

(*) Banco X:

Acciones de Pedro $=(100.77)(0.4769)=48.06$ Valor $=(48.06)(79.35)=3,813.56$

Acciones de Julio $=(100.77)(0.3959)=39.89$ Valor $=(39.89)(79.35)=3,165.27$

Acciones de Marc. $=(100.77)(0.1272)=12.82$ Valor $=(12.82)(79.35)=1,017.26$

(**) Empresa 3:

Acciones de Pedro $=(162.23)(0.4769)=77.37 \quad$ Valor $=(77.37)(21.63)=1,673.51$

Acciones de Julio $=(162.23)(0.3959)=64.23$ Valor $=(64.23)(21.63)=1,389.29$

Acciones de Marc. $=(162.23)(0.1272)=20.63$ Valor $=(20.63)(21.63)=446.23$ 
Estos porcentajes coinciden con las ponderaciones que recibe cada empresa en la cartera de mercado, $\omega_{\mathrm{jm}}{ }^{10}$. Por este motivo, esta estrategia se denomina estrategia pasiva (mantener la cartera de mercado).

Finalmente, en este caso, el número total de acciones de cada empresa que demandan en agregado Pedro, Julio y Marcelo es exactamente igual al número de acciones en circulación que tiene cada empresa (en total 1,328.63 millones de acciones). En otras palabras, el mercado se vacía al invertir cada uno de ellos precisamente la proporción, $\omega_{\text {im }}$, y no otra en cada activo $(21.44 \%$ en el Banco X; $56.42 \%$ en la Empresa $1 ; 13.15 \%$ en la Empresa 2 y el $9.40 \%$ en la Empresa 3). La cartera tangente, aquella cartera escogida por los tres inversores, es necesariamente la cartera de mercado.

La conclusión es que, una vez más, la cartera tangente demandada por los tres inversores debe ser la cartera de mercado.

Finalmente, para expresar el Modelo Sharpe-Lintner (CAPM deSharpey Lintner), se sustituye en la ecuación:

$$
E\left(R_{\mathrm{r}}\right)=\mathrm{r}+\left[\mathrm{E}\left(\mathrm{R}_{\mathrm{T}}-\mathrm{r}\right] \boldsymbol{\beta}_{\mathrm{j} \mathrm{T}}\right.
$$

La cartera tangente $T$ por la cartera de mercado, obteniéndose la expresión:

$$
E\left(R_{1}\right)=r+\left[E\left(R_{m}-r\right] \beta_{j m ; i=1, \ldots, N}\right.
$$

Esta ecuación es el denominado "modelo de valoración de activos con cartera de mercado o CAPM de Sharpe y Lintner", donde el rendimiento esperado de la cartera de mercado esta dada por la expresión:

$$
\mathrm{E}\left(\mathrm{R}_{\mathrm{m}}\right)=\sum_{j=1}^{N} \omega j m \mathrm{E}\left(\mathrm{R}_{\mathrm{i}}\right)
$$

$\mathrm{Y}$, el riesgo beta de cada activo viene dado por la expresión:

$$
\beta \mathrm{jm}=\frac{\operatorname{cov}(R j, R m)}{\sigma_{m}^{2}}
$$

El coeficiente beta $(\beta)$ relevante estará siempre basado en la covarianza entre el rendimiento del activo en cuestión (activo i) y el de la cartera de mercado. Mide la contribución del activo $j$ al riesgo (varianza) de dicha cartera eficiente.

También, por otro lado, el CAPM representa, asimismo, la línea de mercado de activos (LMA) una vez que se reconoce a la cartera tangente como la cartera de mercado. En definitiva, la LMA contiene la relación entre el rendimiento esperado de un activo $j$ y su riesgo sistemático descrito mediante su coeficiente $\beta$.

Este modelo (CAPM de Sharpe y Lintner) puede entenderse como el rendimiento esperado (requerido) por cualquier inversor interesado en comprar el activo j. Así, el inversor exigiría al menos el tipo de interés r, que ofrece un activo seguro. Pero, además, exigiría una compensación adicional pot soportar el riesgo de invertir en el activo j. Dicha prima tiene, según éste modelo, los siguientes componentes:

1) Por un lado, la beta del activo jo, lo que es lo mismo, la contribución del riesgo de $j$ al riesgo (varianza) de la cartera de mercado; y

$10 \omega_{\text {in }}$ es la ponderación de cada activo jen la cartera de mercado. Una cartera de mercado es aquella que incluye todos los activos financieros que existen en el mercado, ponderando cada uno de ellos como:

$\omega_{\mathrm{im}}=\frac{P j . n c j}{\sum_{j=1}^{N} P j . n c j} ; \mathrm{j}=1, . ., \mathrm{N} \quad \mathrm{n}_{\mathrm{ci}}$ es el número total de activos disponibles en la economía. 
2) Por otro, la prima de riesgo del mercado, entendida como una buena aproximación de la cartera de mercado, el Índice General de la Bolsa de Valores.

Es importante resaltar que, dado que la cartera de mercado es eficiente, dicha prima de riesgo debe ser positiva. Así, el CAPM indica que el rendimiento esperado de cualquier activo es una función lineal y positiva del riesgo beta respecto a la cartera de mercado.

Por último, cuando los agentes económicos tienen expectativas homogéneas sobre el conjunto de oportunidades de inversión a la que se enfrentan, el vaciado de mercado permite identificar con precisión la cartera de activos inciertos óptima para todos los inversores, ésta es la cartera de mercado.

De todo lo anterior, se puede concluir que el CAPM sigue siendo el modelo de valoración más ampliamente utilizado para estimar los valores óptimos de los títulos de las empresas para su financiación.

\section{REFERENCIAS}

ALVAREZ,M.I.yDUISBERG,M.A. Cálculo de las tasas de costo de capital para proyectos en empresas en marcba. Santiago, CIADE, Universidad de Chile, 2000.

ALIBER, R. Riesgo de Cambio y Financiación en la Empresa. Madrid, España, Pirámide, 1983.

ARELLANO, Ignacio. "Cómo hacer frente a los riesgos de futuros financieros". En: Revista Estrategia financiera N. ${ }^{\circ} 48,1990$.

ARAGONÉS,José. Economia Financiera Internacional. Madrid, Edit. Pirámide, 1990.

BOREL, Máximo y ROA, Alfonso. Los mercados de futuros financiero. Barcelona, Ariel, 1990.
BREALEY, Richard y MYERS, Stewart. Fundamentos de financiación empresarial. Madrid, McGraw-Hill, 1993.

COSTA, Luis y FONT, Montserrat. Nuevos instrumentos financieros. Madrid, ESIC, 1992.

DIEZ DE CASTRO, Luis y MASCAREÑAS, Juan. "Introducción a la Ingeniería Financiera". En: Revista Contabilidad teoría y práctica. Santiago de Chile, enero-junio, 1988.

Ingeniería Financiera. La Gestión en los Mercados Financieros Internacionales. Madrid, McGraw-Hill, 1994.

EITEMAN, D. y STONEHILL, A. Lasfinanzas de las empresas multinacionales. Willmington, Addinson-Wesley, 1992.

ELTON, Edwin y GRUBER, Martín. Modern porffolio theory and investment analysis. Nueva York, John Wiley, 1991.

FONT, Montserrat. "Nuevos instrumentos paralagestión financiera dela empresa". En: Revista Empresay sociedad. N. ${ }^{\text {os }}$ 5-6, 1989.

FREIXAS, Xavier. Futuros financieros. Madrid, Alianza, 1990.

GORDON, M.J. y SHAPIRO, E. “Capital Equipment Analysis: The Required Rate of Profit". En:Management science. Octubre de 1956.

HERRERA, Beatriz. "Las Decisiones de Financiamiento de Capital". En: Revista Quipukamayoc. Lima, UNMSM, N. ${ }^{\circ} 24$, 2005.

"Las Decisiones de Inversión de los Individuos". En: Revista Quipukamayoc. Lima, UNMSM, N. ${ }^{\circ} 25$ (en prensa), 2006.

LAMOTHE, Prosper. Opciones financieras. Madrid, McGraw-Hill, 1993. 
MARÍN,J.M. y RUBIO, G. Economia financiera. Barcelona, Antoni Bosch, 2001.

MASCAREÑAS, Juan. "La Estructura Temporal de los Tipos de Interés". En: Revista Actualidad financiera N. ${ }^{\circ}$ 19, 1991.

"La Gestión de Carteras de Renta Fija. El Cálculo del Rendimiento". En: Revista Actualidad financiera. N. ${ }^{\circ} 20,1991$.

MASCAREÑAS, J. y LEJARRIAGA, G. Análisis de la estructura de capital de la empresa. Madrid, Eudeva, 1993.

MASCAREÑAS, J. y LÓPEZ, J. Renta fja y fondos de inversión. Madrid, Pirámide, 1997.
MODIGLIANI, Franco y MILLER, Merton. "The Cost of Capital, Corporation Finance and the Theory of Investment". En: American review N. ${ }^{\circ}$ 48, 1958.

ROSS, S.; WESTERFIELD, R. y JAFFE, J. Finanzas corporativas. $5 .^{\mathrm{a}}$ ed. México, McGraw-Hill, 2000.

SHARPE, W.; ALEXANDER, G. yBAILEY, J. Investments. Prentice Hall International, 1995.

SUÁREZ SUÁREZ, Andrés. Decisiones óptimas de inversión y financiación en la empresa. Madrid, Pirámide, 1993.

VALERO, Francisco. Opciones en instrumentos financieros. Barcelona, Ariel, 1988. 